\title{
Role of Mu-Opioid Receptor in Nociceptive Modulation in the Anterior Cingulate Cortex of Rats with Inflammatory Pain
}

\author{
Wang $\mathrm{LL}^{1}$, Hou $\mathrm{KS}^{1}$, Wang $\mathrm{HB}^{1}$, Fu FH ${ }^{1^{*}}$, Yu LC $\mathrm{L}^{1,2^{*}}$
}

${ }^{1}$ Key Laboratory of Molecular Pharmacology and Drug Evaluation, School of Pharmacy, Collaborative Innovation Center of Advanced Drug Delivery System and Biotech Drugs in Universities of Shandong, Yantai University, Ministry of Education, Yantai, P.R. China; ${ }^{2}$ Neurobiology Laboratory, College of Life Sciences, Peking University, Beijing, P.R. China

\begin{abstract}
Objective: The present study was performed to explore the role of mu opioid receptor (MOR) in nociceptive modulation in anterior cingulate cortex (ACC) of rats with inflammatory pain.

Methods: To set up an inflammatory pain model, rats received a subcutaneous injection of $0.1 \mathrm{ml}$ of $2 \%$ carrageenan into the left hind paw. The hind paw withdrawal latency (HWL) to thermal and mechanical stimulation, by hot plate and Randall Selitto Test respectively, was used to evaluate the rat's responses to noxious stimulation. The influence of inflammatory pain on MOR mRNA level and MOR expression in ACC were assayed by Reverse Transcription Polymerase Chain Reaction (RT-PCR) and western blot.

Results: We found that intra-ACC administration of morphine-induced significant antinociceptive effects in a dosedependent manner in rats with inflammatory pain. Furthermore, the antinociceptive effects induced by morphine were attenuated by intra-ACC injection of the opioid receptor antagonist naloxone, indicating involvement of opioid receptor in nociceptive modulation in ACC in rats with inflammatory pain. Moreover, intra-ACC administration of the MOR antagonist $\beta$-funaltrexamine ( $\beta$-FNA) attenuated the morphine-induced antinociception significantly in rats with inflammatory pain. Interestingly, we found that there were significant decreases in MOR mRNA level and MOR expression in ACC in rats with inflammatory pain compared with intact rats tested by RT-PCR and western blot, indicating that there is a down-regulation in MOR expression in rats with inflammatory pain, which support our above results that morphine-induced antinociception was lower in rats with inflammatory pain than that in normal rats.
\end{abstract}

Conclusion: These findings suggest that MOR plays an important role in nociceptive modulation in ACC in rats with inflammatory pain and there is a down-regulation in MOR expression in rats with inflammatory pain.

Keywords: Anterior cingulate cortex; Hind paw withdrawal latency; Inflammatory pain; Morphine-induced antinociception; Mu opioid receptor (MOR); MOR expression; MOR mRNA level; Nociceptive modulation

\section{INTRODUCTION}

Lots of studies have demonstrated that anterior cingulate cortex (ACC) is involved in acute and chronic pain [1-6]. Ikeda and her colleagues found that the activation of astrocytes in the ACC plays a crucial role in the development of negative emotions and LTP during pain hypersensitivity after peripheral inflammation [4]. Zhao and her colleagues found that calmodulin-stimulated adenylyl cyclases (AC), AC-1 and/or AC-8, were crucial in mediating the long-lasting enhanced presynaptic transmitter release in the ACC of mice with chronic pain [5]. Zhuo discussed and reviewed that the possible molecular mechanisms for long-term plasticity within the ACC, and it is hypothesized that potentiation of excitatory responses within the ACC contributes to chronic pain and painrelated mental disorders [6]. Recently, a study in our laboratory demonstrated that galanin induced antinociception in ACC in rats with inflammatory pain, and there were plastic changes

*Correspondence to: Fu FH, Key Laboratory of Molecular Pharmacology and Drug Evaluation, School of Pharmacy, Collaborative Innovation Center of Advanced Drug Delivery System and Biotech Drugs in Universities of Shandong, Yantai University, Ministry of Education, Yantai, P.R. China, Tel: 13806385690; E-mail: fufh@ytu.edu.cn

Yu LC, Neurobiology Laboratory, College of Life Sciences, Peking University, Beijing 100871, P R China, Tel:13552867766; E-mail: yulc@pku.edu.cn

Received: February 28, 2019; Accepted: March 21, 2019; Published: March 26, 2019

Citation: Wang LL, Hou KS, Wang HB, Fu FH, Yu LC (2019) Role of Mu-Opioid Receptor in Nociceptive Modulation in the Anterior Cingulate Cortex of Rats with Inflammatory Pain. J Clin Exp Pharmacol 9:259. doi: 10.35248/2161-1459.19.9.259

Copyright: (C) 2019 Wang LL, et al. This is an open-access article distributed under the terms of the Creative Commons Attribution License, which permits unrestricted use, distribution, and reproduction in any medium, provided the original author and source are credited. 
in the expression of galanin and galanin receptor 2 in rats with inflammatory pain [7].

Opioid peptides or anti-opioid peptides exert analgesic or antianalgesic effect through binding to their corresponding receptors. High levels of opioid receptors are expressed in rostral regions of the ACC [8]. ACC has one of the highest densities of opioid receptors in the CNS and it has been implicated in acute and chronic pain responses [9]. There exist 3 major opioid receptor subtypes, mu, kappa and delta, in the CNS, the opioid drugs functions, such as analgesia, rewarding and addiction. Opiates, especially the agents acting on mu opioid receptor (MOR), serve as the most effective analgesics for clinical patients with severe pain [10]. It has been demonstrated that MOR presents throughout the cingulate cortex [9]. Zheng has reported that chronic peripheral inflammation significantly enhanced the presynaptic glutamate release in the ACC, which was significantly suppressed by activation of MOR [11].

In clinic, inflammatory pain is one of the most common chronic pain conditions. The present study was performed to explore the influence of inflammatory pain on MOR expression as well as the morphine-induced antinociception in ACC in rats with inflammatory pain tested by pharmacological and biological methods.

\section{MATERIALS AND METHODS}

\section{Animals}

Sprague-Dawley rats, male, weighing 220 to 260 g, 6-7 weeks, were provided by Experimental Animal Center of Luye Pharmaceutical Company, Yantai, China, with certificate number 20170018. The rats were housed in the normal day/night cycle and allowed free access to food and water, and the room temperature was kept at 20 $\pm 2^{\circ} \mathrm{C}$. Before the experiment, all rats were accustomed to the test condition for three days.

All experiments relative to animals were performed according to the Guidelines of the International Association for the Study of Pain [12] and the Guidelines for the Care and Use of Laboratory Animals of Yantai University and were approved by Laboratory Animal Ethics Committee of Yantai University, with the authorization number YT-YX-1817.

\section{To plant the guide cannula for intra-ACC injection}

The surgical procedure was as follows. Firstly, rats were anaesthetized by injecting intraperitoneally with pentobarbital sodium produced by Xudong Chemical Factory, Beijing, China, at a dosage of 50 $\mathrm{mg} / \mathrm{kg}$, then mounted on the stereotaxic frame. For injection, the ACC, $1.6 \mathrm{~mm}$ anterior to Bregma; $0.7 \mathrm{~mm}$ lateral to midline; 2.0 $\mathrm{mm}$ ventral to the surface of skull was recognized according to Paxinos and Watson [13] and a stainless steel guide cannula with $0.8 \mathrm{~mm}$ outer diameter was directed to the ACC, and fixed to the skull by dental acrylic. Thereafter the rats were maintained and recovered at least for 3 days.

\section{Inflammatory pain model and intra-ACC injection}

The left hind paw of the rat was subcutaneously injected with 0.1 $\mathrm{ml}$ of $2 \%$ carrageenan ( $2 \mathrm{mg}$ in $0.1 \mathrm{ml}$ saline, Sigma) to make an inflammatory pain model. On the experiment day, a stainless steel needle was directly inserted into the guide cannula with $1.5 \mathrm{~mm}$ beyond the tip of the latter. One microliter of drug or vehicle was thereafter infused into the ACC lasted for $1 \mathrm{~min}$. The injection needle was left in the site for 1 min after each injection before removal.

To all animals, before and after the injection of carrageenan, the hind paw withdrawal latency (HWL) to noxious thermal and mechanical stimulation was assessed by the hot-plate test and the Randall Selitto Test [14].

\section{Nociceptive tests}

The HWLs were measured as described previously [7,14-16]. To mechanical stimulation, HWL was observed by the Randall Selitto Test (Ugo Basile, Type 7200, Italy) with a wedge-shaped pusher at a loading rate of $30 \mathrm{~g} / \mathrm{s}$ being applied to the dorsal surface of the hind paw. To noxious thermal stimulation, the entire ventral surface of the rat hind paw was placed manually on a hot plate (YLS-6B Intelligent Heat Panel Instrument, Jinan Yiyan Science \& Technology Development Co., Ltd., Jinan, China) at the temperature of $52 \pm 0.2^{\circ} \mathrm{C}$. The time to hind paw withdrawal was measured and expressed in seconds, and referred to as the HWL to mechanical stimulation or thermal stimulation. Each rat was operated with both stimulations.

The HWLs, before intra-ACC injection, was tested three times as the basal HWLs. The HWLs after drug injection were expressed as percentage changes of the basal HWL for every rat (\% changes to the HWL). In order to diminish the damage, there was a cut-off limit of $15 \mathrm{~s}$ and the operating time for every measuring the HWL was no more than $2 \mathrm{~min}$.

\section{Reverse transcription polymerase chain reaction for testing mRNA}

The rats with inflammatory pain $(n=3)$ and normal rats as a control $(n=3)$ received overdose of $10 \%$ trichloroacetaldehyde monohydrate. The brains were removed and frozen on dry ice. Then tissues of ACC were dissected on ice and then stored at $-80^{\circ} \mathrm{C}$. Reverse Transcription Polymerase Chain Reaction (RT-PCR) was carried out as described in our published paper $[16,17]$. Tissues were lysed in TRIzol (CoWin Biosciences, Beijing, China), and total RNA was prepared. After reverse transcription using a first-strand cDNA synthesis kit (CoWin Biosciences, Beijing, China), the cDNA was subjected to RT-PCR assays using the 7500 Fast RT-PCR System (Applied Biosystems $^{\mathrm{TM}}$, Thermo Fisher, America). Forward and reverse primer sequences for the experiments were rat MOR were as follows: sense 5'-TCTAATGTATTGTCTGGTTTGCCGTATTG-3' and antisense 5'-AACTCTTCATGTAAGGTGACTAGGTGCTTC-3'; rat GAPDH: sense 5'-GACCACCCAGCCCAGCAAGG-3' and antisense 5'-TCCCCAGGCCCCTCCTGTTG-3'. The unigene expression levels were calculated with the 2- $\Delta \Delta \mathrm{CT}$ method [18]. RT-PCR data are presented as the normalized ratio of the target gene relative to the GAPDH control gene using $\triangle \mathrm{CT}$.

\section{Using western blot to test the MOR expressions}

For Western blot, rats with inflammatory pain $(n=3)$ and normal rats as a control $(n=3)$ received overdose of $10 \%$ trichloroacetaldehyde monohydrate and the brain tissue of ACC was removed and dissected on ice, and then frozen at $-80^{\circ} \mathrm{C}$ immediately. The brain tissue samples were homogenized in RIPA lysis buffer (Beyotime Institute of Biotechnology, Shanghai, China) containing phenylmethanesulfonyl fluoride (Beyotime Institute of 
Biotechnology, Shanghai, China) and centrifuged at $12,000 \mathrm{rpm}$ for $15 \mathrm{~min}$ at $4^{\circ} \mathrm{C}$. The supernatant was collected and measured by BCA Protein Assay Kit (Beyotime Institute of Biotechnology, Shanghai, China). Whole protein extracts $(80 \mu \mathrm{g})$ of ACC samples were subject to $10 \%$ SDS-polyacrylamide gel electrophoresis. Then the proteins were transferred to polyvinylidene fluoride membranes (Millipore, MA, USA) for $1 \mathrm{~h}$ at $106 \mathrm{~V}$. The membranes were incubated in blocking solution (5\% non-fat milk in Tris-buffered saline containing $0.1 \%$ Tween-20, TBST) for $2 \mathrm{~h}$ (room temperature), and sequentially in TBST (Tris- $\mathrm{HCl}, \mathrm{NaCl}$, Tween-20) containing primary antibodies to the polyclonal rabbit anti-MOR antibody (1:1000; ab10275, Abcam, Cambridge, UK), or beta-actin antibody (1:1000; AA1128, Beyotime Institute of Biotechnology, Shanghai, China) overnight $\left(4^{\circ} \mathrm{C}\right)$. The membranes were washed 3 times with TBST for $10 \mathrm{~min}$ each and then probed with HRP-conjugated goat anti-rabbit secondary antibody (1:1000; ab6721, Abcam, Cambridge, UK), HRP-conjugated goat antimouse secondary antibody (1:1500; A0216, Beyotime Institute of Biotechnology, Shanghai, China) for $1 \mathrm{~h}$ (room temperature). The membranes were washed 3 times with TBST for 10 min each again. The brands were visualized by enhanced chemiluminescence detection reagents (Beyotime Institute of Biotechnology, Shanghai, China) and imaged using ChampChemi (Beijing SageCreation Science Co., Ltd) and quantified using SageCapture software (Beijing SageCreation Science Co., Ltd).

\section{Statistical analysis}

The location of the tip of the injection needle was verified and all the injection points were in ACC of rats. All the tips of the injection needle are within the ACC. Data from the experiment were expressed as mean \pm S.E.M.

Statistical difference between groups was determined by two-way analysis of variance (ANOVA) for repeated measurements (Fleft/ left is the F value of the two groups: the left HWL of one group compared with the left HWL of another group) or Student's t-test (two-tailed). ${ }^{*} \mathrm{P}<0.05,{ }^{* *} \mathrm{P}<0.01$ and ${ }^{* *} \mathrm{P}<0.001$ were considered as significant differences.

\section{Chemicals for intra-ACC injection}

Solutions for intra-ACC administration were prepared with sterilized saline, each with a volume of $1 \mu$ containing: (1) 1,5 , or $10 \mu \mathrm{g}$ of morphine (morphine hydrochloride; Shenyang First Pharmaceutical Factory, China); (2) $20 \mu \mathrm{g}$ of naloxone (naloxone benzoylhydrazone; Tocris, UK); (3) $10 \mu \mathrm{g}$ of $\beta$-funaltrexamine ( $\beta$-Funaltrexamine hydrochloride, घ-FNA; Tocris, UK).

\section{RESULTS}

\section{Morphine-induced antinociception in ACC of rats with inflammatory pain}

To set up an inflammatory pain model, rats received a subcutaneous injection of $0.1 \mathrm{ml}$ of $2 \%$ carrageenin $(2 \mathrm{mg}$ in 0.1 $\mathrm{ml}$ saline, Sigma) into the left hind paw. The HWLs to noxious thermal and mechanical stimulation were assessed before and after the injection of carrageenan measured by the hot-plate test and Randall Selitto Test. As shown in Figure 1, A and B, HWLs to thermal stimulation; $\mathrm{C}$ and D, HWLs to mechanical stimulation. Left HWL: A and C; right HWL: B and D. As shown in Figure 1A and $1 \mathrm{C}$, the left HWLs were significantly decreased at $3 \mathrm{~h}$ after carrageenin injection (Hot-plate Test: $\mathrm{p}<0.001, \mathrm{t}=9.53$; Randall Selitto Test: $\mathrm{p}<0.001, \mathrm{t}=6.45$; two-tailed student's $\mathrm{t}$-test), and the left HWLs were significantly decreased at $4 \mathrm{~h}$ after carrageenin injection (Hot-plate Test: $\mathrm{p}<0.001, \mathrm{t}=15.66$; Randall Selitto Test: $\mathrm{p}<0.001, \mathrm{t}=9.07$; two-tailed student's t-test). As shown in Figure 1B and $1 \mathrm{D}$, there were no significant changes in right HWLs at $3 \mathrm{~h}$ after carrageenin injection (Hot-plate Test: $p=0.52, t=0.66$; Randall Selitto Test: $\mathrm{p}=0.47, \mathrm{t}=0.75$; two-tailed student's t-test), and there were no significant changes in right HWLs at $4 \mathrm{~h}$ after carrageenan
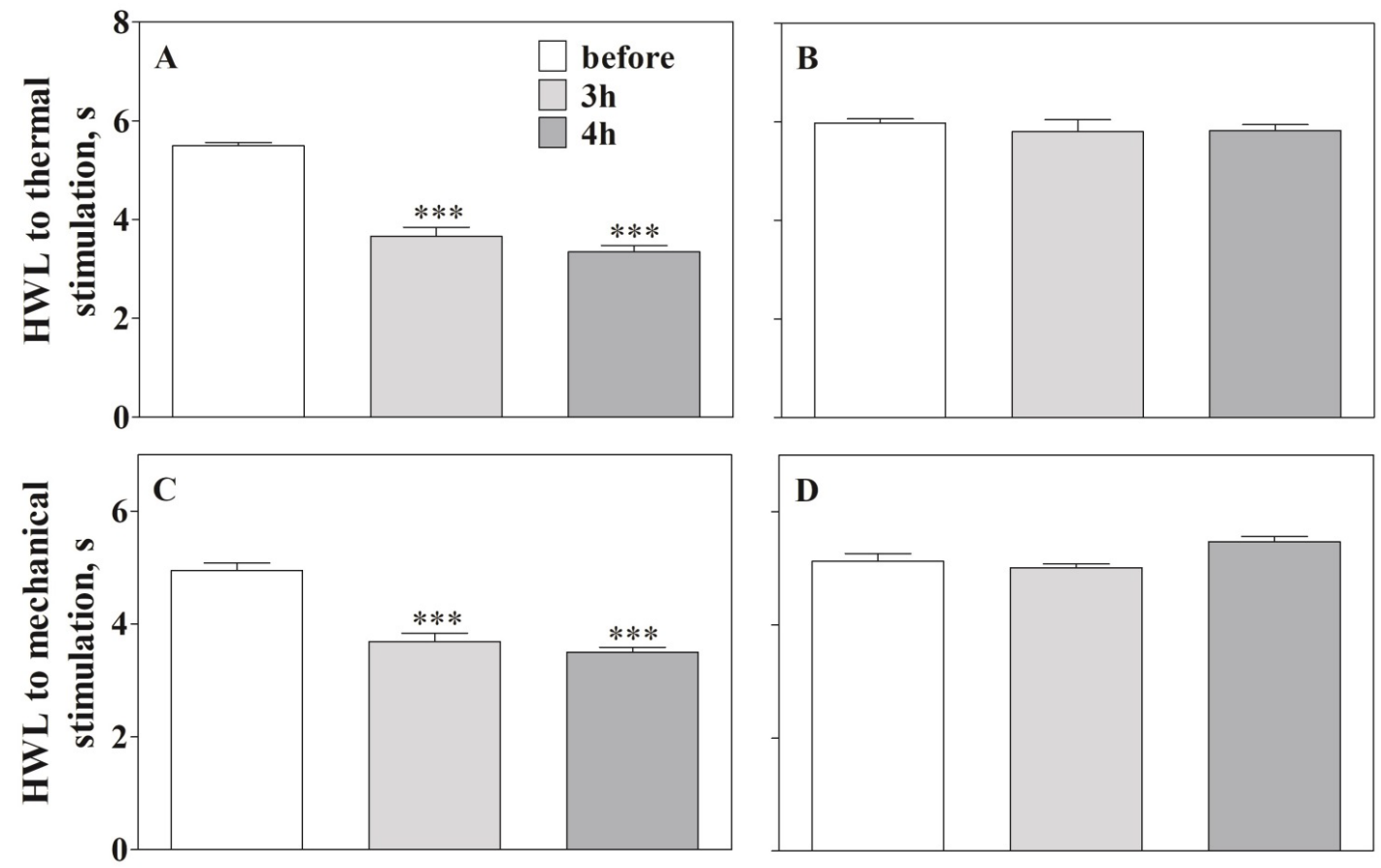

Figure 1: Basal HWLs before and after carrageenan injection. These results exhibited that there was inflammatory pain in left paw of the rats during $3 \mathrm{~h}$ to $4 \mathrm{~h}$ after carrageenan injection. A and B, HWLs to thermal stimulation; C and D, HWLs to mechanical stimulation. Left HWL: A and C; right HWL: $\mathrm{B}$ and D. All values are expressed as Mean \pm SEM. Data was analyzed by Student's t-test (two tails). ${ }^{* *} \mathrm{p}<0.001$ as compared to control group. HWL, hindpaw withdrawal latency. 
injection (Hot-plate Test: $\mathrm{p}=0.32, \mathrm{t}=1.04$; Randall Selitto Test: $p=0.06, t=2.06$; two-tailed student's t-test). These results exhibited that there was inflammatory pain in the left paw of the rats during $3 \mathrm{~h}$ to $4 \mathrm{~h}$ after carrageenan injection.

To observe the influence of morphine in ACC on nociceptive responses in rats with inflammatory pain, four groups of rats with inflammatory pain were received intra-ACC injection of $1 \mu 1$ of $0.9 \%$ saline (as control, $n=7)$, or $1 \mu \mathrm{g}(\mathrm{n}=7), 5 \mu \mathrm{g}(\mathrm{n}=7)$ or $10 \mu \mathrm{g}$ $(n=7)$ of morphine. The results showed that the HWLs to thermal and mechanical stimulations increased significantly after intraACC injection of $1 \mu \mathrm{g}$ of morphine (Hot-plate Test: Fleft/left=2.85, $\mathrm{p}=0.10$; Fright/right $=2.41, \mathrm{p}=0.12$. Randall Selitto Test: Fleft/ left=12.48, p<0.01; Fright/right=1.18, p=0.28), $5 \mu \mathrm{g}$ of morphine (Hot-plate Test: Fleft/left=65.26, p<0.001; Fright/right=95.43, $\mathrm{p}<0.001$; Randall Selitto Test: Fleft/left $=109.64$, $\mathrm{p}<0.001$; Fright/ right $=73.54, \mathrm{p}<0.001$ ), and $10 \mu \mathrm{g}$ of morphine (Hot-plate Test: Fleft $/$ left $=110.60, \mathrm{p}<0.001 ;$ Fright $/$ right $=71.56, \mathrm{p}<0.001$; Randall Selitto Test: Fleft/left=141.52, $\mathrm{p}<0.001 ;$ Fright/right=161.17, $\mathrm{p}<0.001$ ) compared with the control group (Figure 2). The results demonstrated that intra-ACC injection of morphine induced significant antinociceptive effects in rats with inflammatory pain in a dose-dependent manner.

\section{MOR mediated the morphine-induced antinociception in $\mathrm{ACC}$ in rats with inflammatory pain}

Two groups of rats with inflammatory pain received the intra-ACC injection $10 \mu \mathrm{g}$ of morphine, followed $5 \mathrm{~min}$ later by intra-ACC injection of $20 \mu \mathrm{g}(\mathrm{n}=6)$ of the opioid receptor antagonist naloxone, or $1 \mu \mathrm{l}$ of $0.9 \%$ saline as a control $(n=6)$. Another group of rats with inflammatory pain $(n=6)$ received an intra-ACC injection of $1 \mu 1$ of $0.9 \%$ saline, followed 5 min later by intra-ACC injection of 20 $\mu \mathrm{g}$ of naloxone.

The results showed that after intra-ACC injection of morphine, there was a marked increase in HWLs to thermal and mechanical stimulation in rats with inflammatory pain. The increased HWLs to thermal and mechanical stimulation were deceased significantly after intra-ACC administration of naloxone (Hot-plate Test: tleft/ left $=9.28, \mathrm{p}<0.001$, decreased 48\%; tright $/$ right $=13.53, \mathrm{p}<0.001$, decreased 40\%; Randall Selitto Test: tleft/left=19.77, $\mathrm{p}<0.001$, decreased 50\%; tright/right=19.07, p<0.001, decreased 40\%) compared with the control group. There are no marked changes in HWLs to thermal and mechanical stimulation after intra-ACC administration of saline and naloxone in rats with inflammatory pain. These results indicated that opioid receptor was involved in the morphine-induced antinociception in the ACC of rats with inflammatory pain (Figure 3).

It is known that morphine mainly activates MOR in the CNS. To further confirm the involvement of MOR in the morphine-induced antinociception in ACC of rats with inflammatory pain, two groups of rats with inflammatory pain received intra-ACC injection of 10 $\mu \mathrm{g}$ of morphine, followed $5 \mathrm{~min}$ later by intra-ACC injection of 10 $\mu \mathrm{g}$ of the MOR antagonist $\beta$-FNA $(n=6)$, or $1 \mu$ of $0.9 \%$ saline as a control $(n=6)$. Another group of rats with inflammatory pain $(n=6)$ received an intra-ACC injection of $1 \mu \mathrm{l}$ of $0.9 \%$ saline, followed 5 min later by intra-ACC injection of $10 \mu \mathrm{g}$ of $\beta$-FNA.

After intra-ACC injection of morphine, there were marked

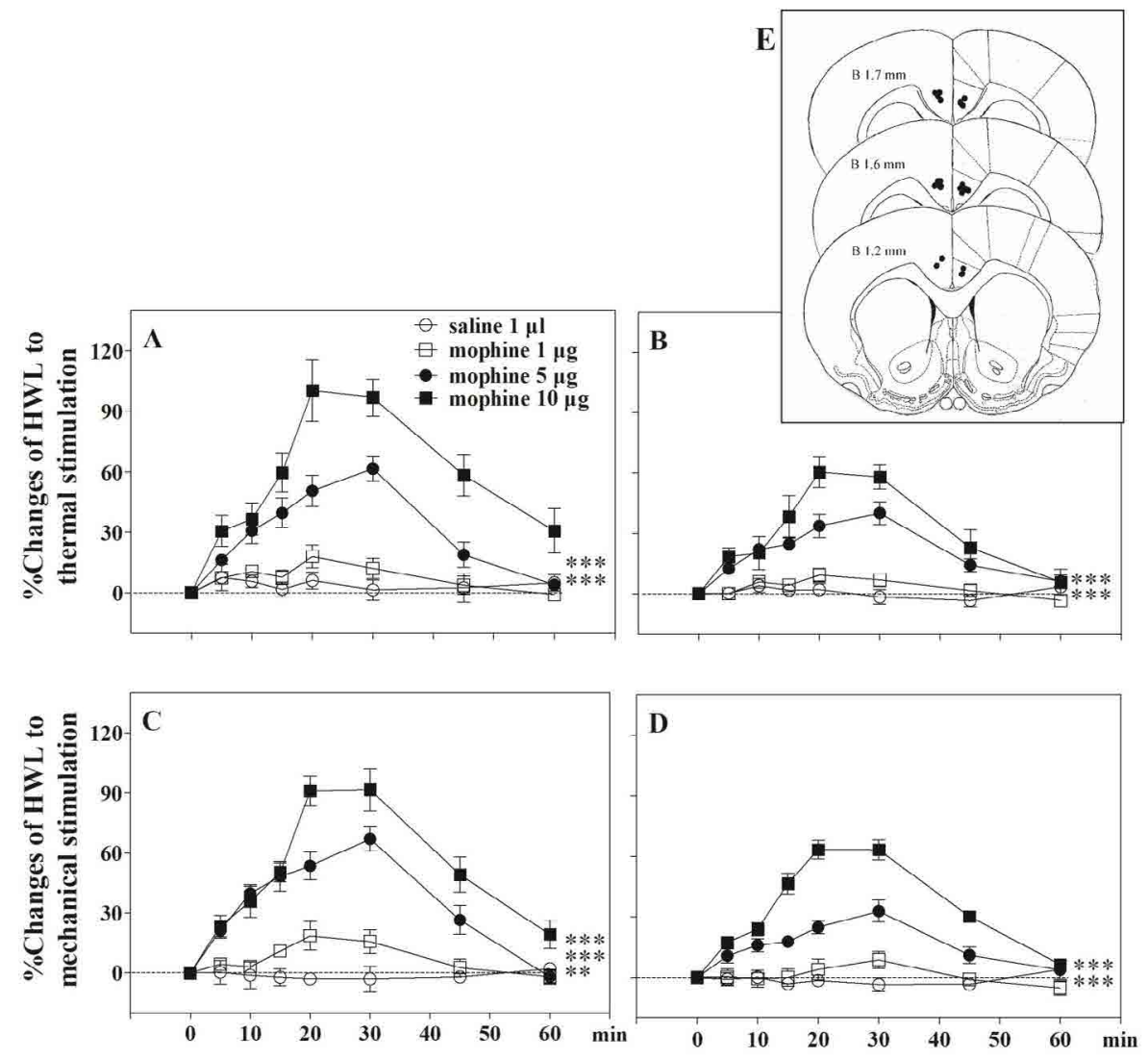

Figure 2: Intra-ACC injection of morphine induced increases in HWLs to thermal and mechanical stimulation in rats with inflammatory pain. Intra-ACC injection of morphine induced significant antinociceptive effects in rats with inflammatory pain in a dose-dependent manner. The HWLs to thermal (A and B) and mechanical stimulation (C and D) increased significantly after intra-ACC injection of morphine in rats with inflammatory pain. Left HWL: A and C; right HWL: B and D. Injection site: E. All values are expressed as Mean \pm SEM. Data was analyzed by Two-way ANOVA followed Bonferroni test. ${ }^{* *} \mathrm{p}<0.01$ and ${ }^{* * *} \mathrm{p}<0.001$. ACC, the anterior cingulate cortex; HWL, hindpaw withdrawal latency. 

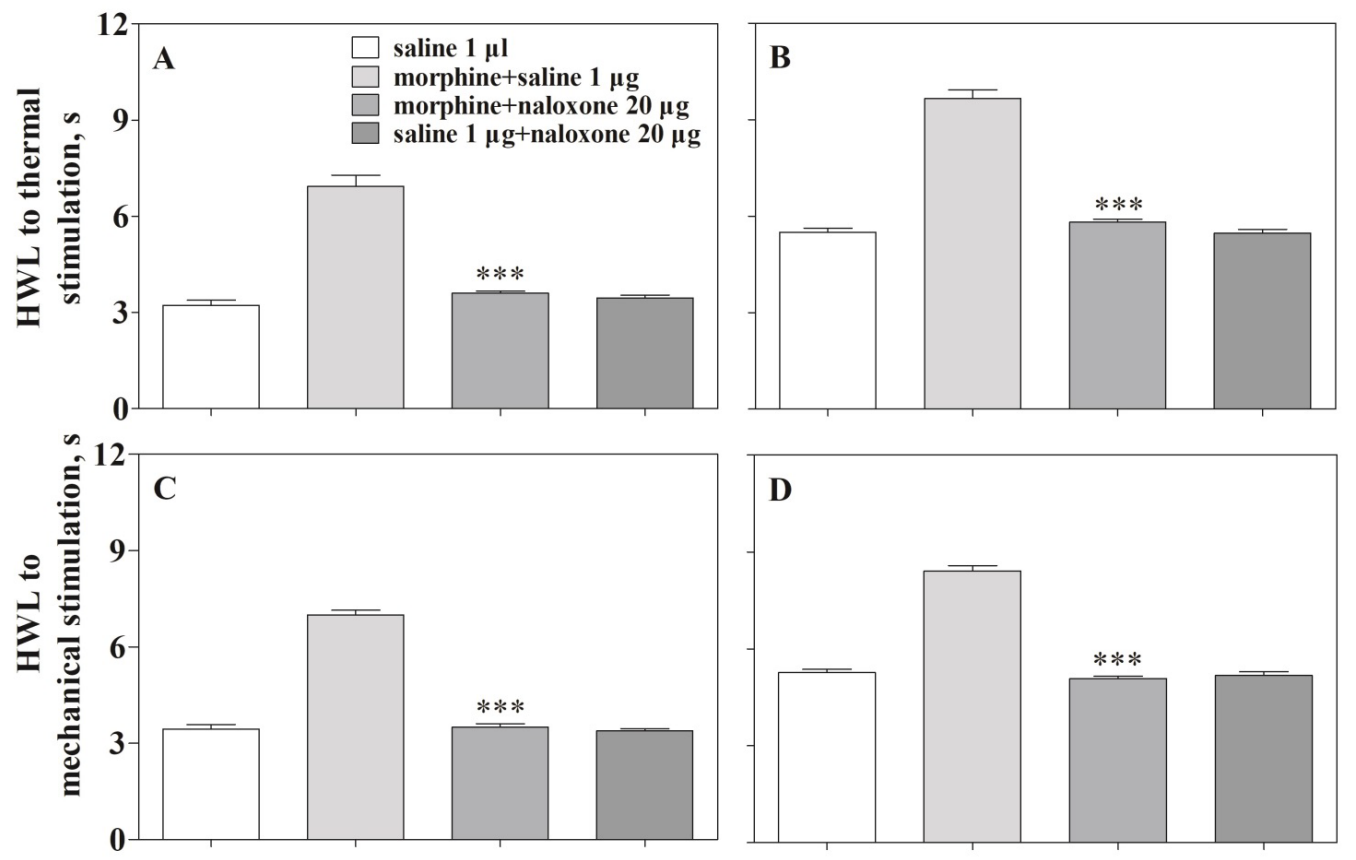

Figure 3: The effect of naloxone on morphine-induced antinociception in ACC in rats with inflammatory pain. The increased HWLs to thermal and mechanical stimulation were deceased significantly after intra-ACC administration of naloxone. A and B, HWLs to thermal stimulation; C and D, HWLs to mechanical stimulation. Left HWL: A and C; right HWL: B and D. Data showed the results $30 \mathrm{~min}$ after drugs injecting morphine and/or naloxone. All values are expressed as Mean \pm SEM. Data was analyzed by Student's t-test (two tails). ${ }^{* * *} \mathrm{p}<0.001$. ACC, anterior cingulate cortex; HWL, hindpaw withdrawal latency.

increases in HWLs to thermal and mechanical stimulation in rats with inflammatory pain. The increased HWLs to thermal and mechanical stimulation were decreased significantly after intra-ACC administration of $10 \mu \mathrm{g}$ of the MOR antagonist $\beta$-FNA (Hot-plate Test: tleft/left=5.88, $\mathrm{p}<0.001$, decreased $25 \%$; tright/right $=10.78$, $\mathrm{p}<0.001$, decreased 37\%; Randall Selitto Test: tleft/left $=10.41$, $\mathrm{p}<0.001$, decreased 35\%; tright/right $=6.48, \mathrm{p}<0.001$, decreased $32 \%)$ compared with the control group. The results also showed that there were no marked influences on the HWLs to thermal and mechanical stimulation after intra-ACC administration of saline and [-FNA in rats with inflammatory pain (Figure 4). These results demonstrated that blockade MOR by $\beta$-FNA inhibited the morphine-induced antinociception significantly, indicating that MOR plays an important role in nociceptive modulation in ACC in rats with inflammatory pain.

\section{Comparison of the morphine-induced antinociception in rats with inflammatory pain and in normal rats}

Furthermore, we compared the morphine-induced antinociception in ACC in normal rats and rats with inflammatory pain. The results showed that there were significant decreases in HWL to noxious thermal stimulation (Hot plate test: tleft/left=12.30, $\mathrm{p}<0.001 ;$ tright $/$ right $=7.33, \mathrm{p}<0.001 ;$ two-tailed student's t-test) and mechanical stimulation (Randall Selitto Test: tleft/left=10.06, $\mathrm{p}<0.001 ;$ tright/right $=6.849, \mathrm{p}<0.001$; two-tailed student's t-test) in rats with inflammatory pain compared to normal rats (Figure 5), indicating that there were decreases in morphine-induced antinociception during inflammatory pain.

\section{Influences of inflammatory pain on MOR expression in $\mathrm{ACC}$}

To explore why there were decreases in morphine-induced antinociception during inflammatory pain, the influence of inflammatory pain on MOR expression was studied. The expression of MOR in ACC and the mRNA levels of MOR in ACC of normal rats $(n=3)$ and rats with inflammatory pain $(n=3)$ were determined by western blot and RT-PCR, and the results showed that there was a significant decrease in expression of MOR ( $<<0.01, t=4.90$, twotailed student's t-test) in ACC in rats with inflammatory pain than that in normal rats (Figure $6 \mathrm{~A}$ and $6 \mathrm{~B}$ ). It was also found that there was a significant decrease in the mRNA level of MOR $(p<0.05$, $\mathrm{t}=2.48$, two-tailed student's t-test) in ACC of rats with inflammatory pain than that in normal rats. The results demonstrate that there was a down-regulation in MOR expression in ACC in rats with inflammatory pain, 3 hours after carrageenan injection. These support our above results that there were decreases in morphineinduced antinociception in ACC in rats with inflammatory pain.

\section{DISCUSSION}

Recent studies have found that there is a close relationship between ACC and inflammatory pain [19]. Thibault and his colleagues reported that brain-derived neurotrophic factor expression is increased in the ACC during inflammation and is sufficient to induce a series of plastic changes such as LTP and extracellular regulated protein kinases phosphorylation leading to cold hypersensitivity, and this could be one such mechanism behind the induction and maintenance of the affective-emotional aspect of pain [20]. Koga and his colleagues demonstrated that presynaptic but not postsynaptic changes occur in the I-aminobutyric acid system during early phases in this chronic inflammatory pain model [19].

Carrageenan model is an acute non-specific inflammatory model, which is often used to observe the effects of various drugs or therapies on the acute inflammatory process. It is a classic and ideal routine model for screening anti-inflammatory drugs or therapies. In this experiment, we found that subcutaneous injection of $2 \%$ carrageenan into the left hind paw in rats has successfully induced local inflammatory pain. The left HWLs were 

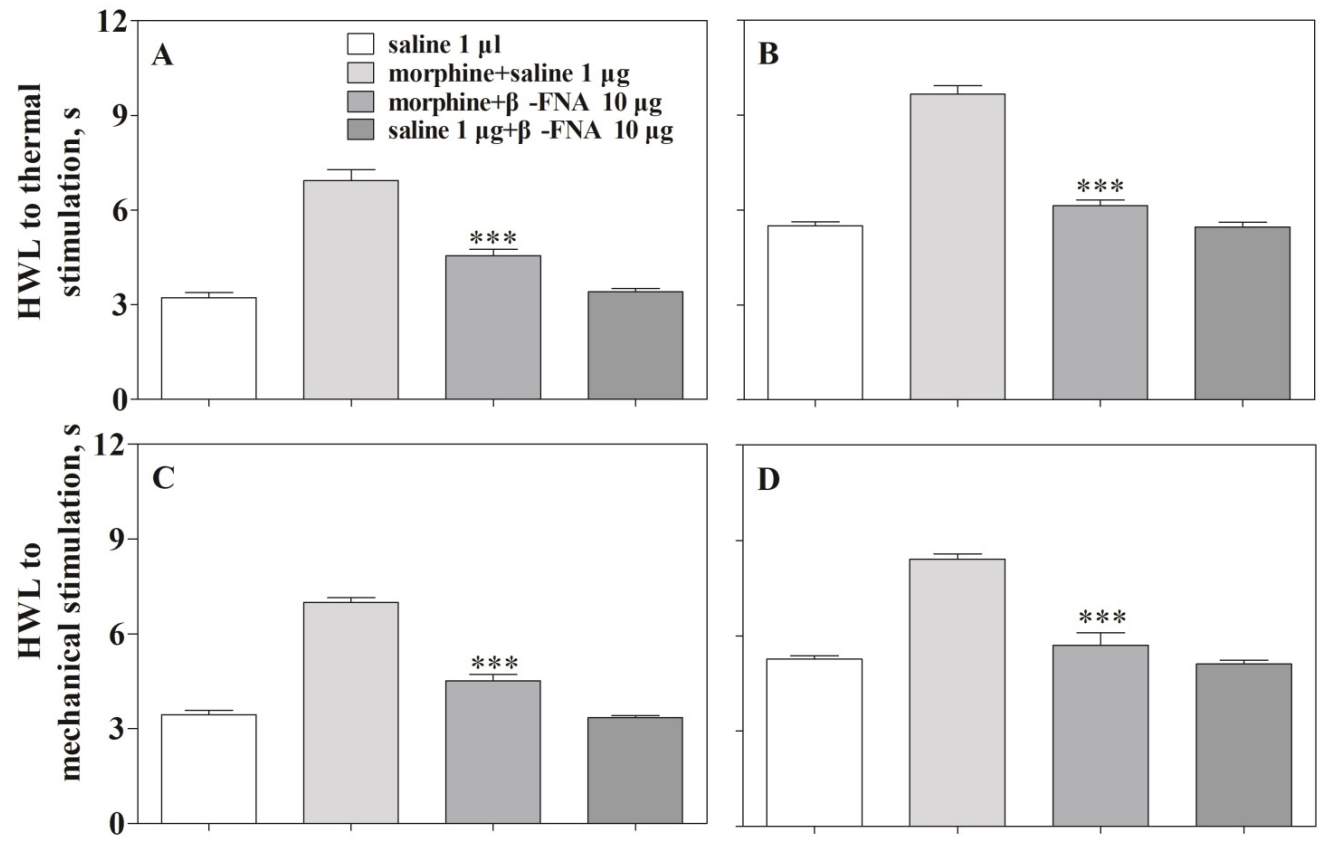

Figure 4: The effect of $\beta$-FNA on morphine-induced antinociception in ACC in rats with inflammatory pain. The increased HWLs to thermal and mechanical stimulation were deceased significantly after intra-ACC administration of $\beta$-FNA. A and B, HWLs to thermal stimulation; C and D, HWLs to mechanical stimulation. Left HWL: A and C; right HWL: B and D. Data showed the results 30 min after injecting morphine and/or $\beta$-funaltrexamine. All values are expressed as Mean \pm SEM. Data was analyzed by Student's t-test (two tails). ${ }^{* * *} \mathrm{p}<0.001$. ACC, anterior cingulate cortex; HWL, hindpaw withdrawal latency. MOR, mu opioid receptor.
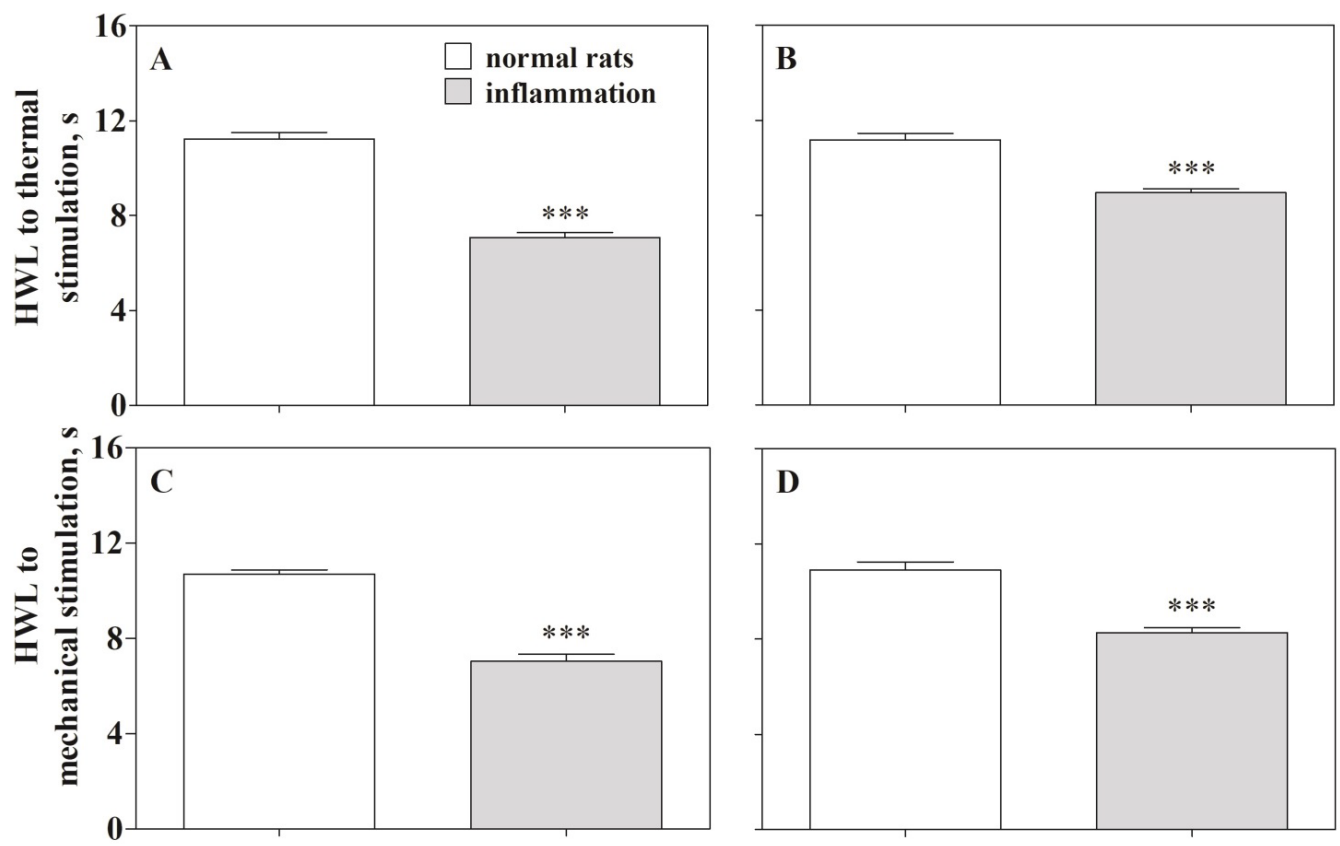

Figure 5: Comparison of the morphine-induced antinociception in rats with inflammatory pain and normal rats. The morphine-induced increases in HWLs to thermal (A and B) and mechanical stimulation (C and D) were decreased significantly in rats with inflammatory pain than that in normal rats. A and B, HWLs to thermal stimulation; C and D, HWLs to mechanical stimulation. All values are expressed as Mean \pm SEM. Data was analyzed by Student's t-test (two tails), ACC, anterior cingulate cortex; HWL, hindpaw withdrawal latency. Student's t-test (two tails). ${ }^{* * *}$ p $<0.001$.

significantly decreased at $3 \mathrm{~h}$ after carrageenin injection, and the left HWLs were significantly decreased at $4 \mathrm{~h}$ after carrageenin injection. There were no significant changes in right HWLs at $3 \mathrm{~h}$ after carrageenin injection, and there were no significant changes in right HWLs at $4 \mathrm{~h}$ after carrageenan injection. These results exhibited that there was inflammatory pain in the left paw of the rats during $3 \mathrm{~h}$ to $4 \mathrm{~h}$ after carrageenan injection.

Our results demonstrated that intra-ACC injection of morphineinduced antinociceptive effects in a dose-dependent manner in rats with inflammatory pain, which suggests that opioid peptides and opioid receptors may involve in antinociception in ACC.

It has been well documented that opiates play an important role in pain modulation in ACC. Currently, Opiates, especially the agents acting on MOR, serve as the most effective analgesics for clinical patients with severe pain [10]. It has been found that both opioid peptides and their receptors are highly expressed in the ACC [21]. There are 3 types of opioid receptors, mu, kappa and delta, in the brain tissue, which plays role need to be elucidated. 
A
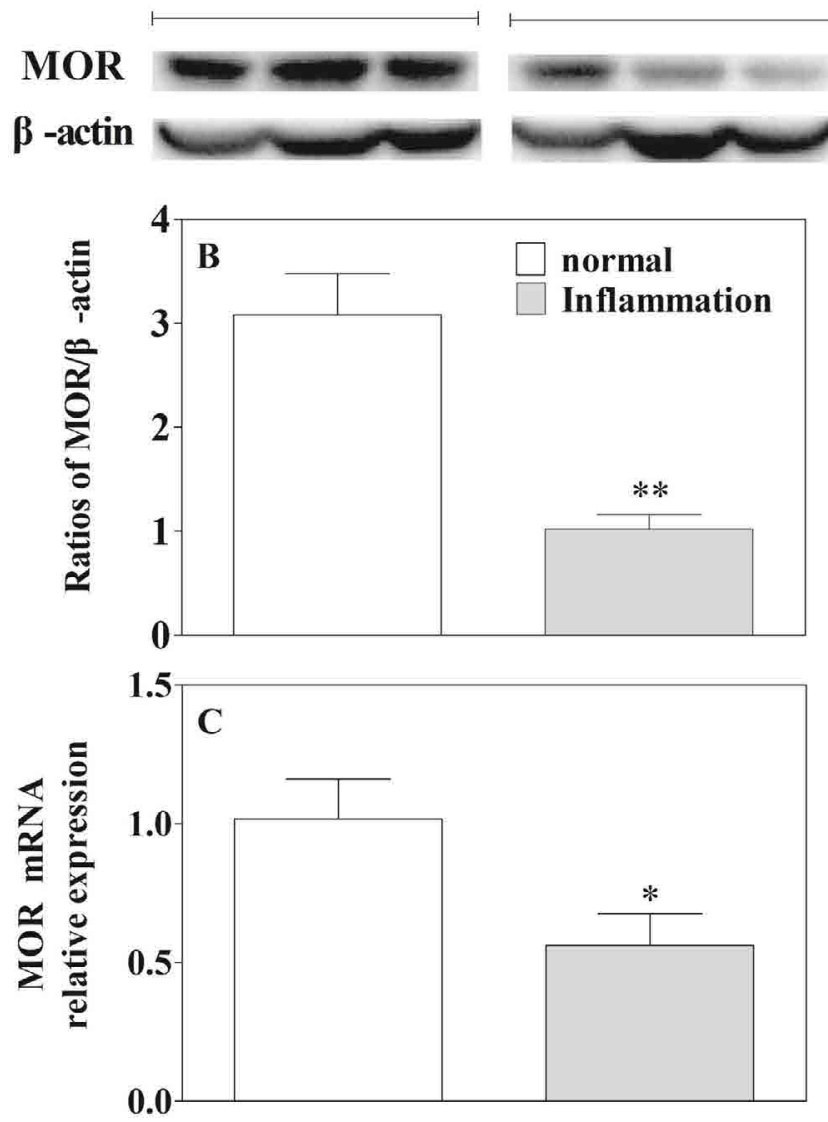

Figure 6: Influences of inflammatory pain on the expression of MOR in ACC. The results demonstrate a down-regulation in MOR expression in ACC in rats with inflammatory pain. A and $B$, the expression of MOR in ACC in normal rats $(n=3)$ and in rats with inflammatory pain $(n=3)$ tested by western blot. C, the MOR mRNA levels in ACC in normal rats and in rats with inflammatory pain tested by RT-PCR. All values are expressed as Mean \pm SEM. Data was analyzed by Student's t-test (two tails). * $\mathrm{p}<0.05$, ** $\mathrm{p}<0.01$. ACC, anterior cingulate cortex; MOR, mu opioid receptor.

Observing the effects of receptor antagonist on chemicals is the means to understand the target of the drug. To investigate whether opioid receptor mediates morphine-induced antinociception in ACC in rats with inflammatory pain, the rats with inflammatory pain were administered with opioid receptor antagonist naloxone. The antinociceptive effects induced by morphine were attenuated significantly by intra-ACC injection of the opioid receptor antagonist naloxone, indicating involvement of opioid receptor in nociceptive modulation in ACC in rats with inflammatory pain. Furthermore, intra-ACC administration of the MOR antagonist [-FNA attenuated the morphine-induced antinociceptive effects, and approximate to that of naloxone. Blockade MOR by I-FNA inhibited the morphine-induced antinociception significantly, further indicating that MOR plays an important role in nociceptive modulation in ACC in rats with inflammatory pain [22].

\section{CONCLUSION}

We found that the morphine-induced antinociception was lower in rats with inflammatory pain than that in normal rats, which suggests that opioid receptors might be changed by making inflammatory pain model. Inflammatory pain may regulate the MOR and subsequently, affect the effects of some analgesics.

Thus the influence of inflammatory pain on MOR expression was investigated. We compared the MOR expressions and MOR mRNA levels between rats with inflammatory pain to normal rats. We found that there was a significant decrease in MOR mRNA level and MOR expression in ACC in rats with inflammatory pain. This means that inflammatory pain results in the down expression of MOR, and in turn, decreasing the antinociceptive effect of morphine. These results indicated that MOR plays an important role in nociceptive modulation in ACC in rats with inflammatory pain. These findings suggest that MOR plays an important role in nociceptive modulation in ACC in rats with inflammatory pain and there is a down-regulation in MOR expression in rats with inflammatory pain.

\section{ACKNOWLEDGEMENTS}

The study is supported by grants from Foundation of Collaborative Innovation Center of Advanced Drug Delivery System and Biotech Drugs in Universities of Shandong (Yantai University), the Graduate Innovation Foundation of Yantai University and the National Natural Science Foundation of China (NSFC 81171043).

\section{CONFLICTS OF INTEREST}

The authors declare no competing financial interests.

\section{REFERENCES}

1. Bliss TV, Collingridge GL, Kaang BK, Zhuo M. Synaptic plasticity in the anterior cingulate cortex in acute and chronic pain. Nat Rev Neurosci. 2016;17:485-496.

2. Du J, Fang J, Wen C, Shao X, Liang Y. The effect of electroacupuncture on PKMzeta in the ACC in regulating anxiety-like behaviors in rats experiencing chronic inflammatory pain. Neural Plast. 2017;2017:3728752.

3. Harris-Bozer AL, Peng YB. Inflammatory pain by carrageenan recruits low-frequency local field potential changes in the anterior cingulate cortex. Neurosci Lett. 2016;632:8-14.

4. Ikeda H, Mochizuki K, Murase K. Astrocytes are involved in longterm facilitation of neuronal excitation in the anterior cingulate cortex of mice with inflammatory pain. Pain. 2013;54:28362843.

5. Zhao MG, Ko SW, Wu LJ, Toyoda H, Xu H. Enhanced presynaptic neurotransmitter release in the anterior cingulate cortex of mice with chronic pain. J Neurosci. 2006;26:8923. 8930.

6. Zhuo M. Molecular mechanisms of pain in the anterior cingulate cortex. J Neurosci Res. 2006;84:927-933.

7. Zhang ML, Fu FH, Yu LC. Antinociception induced by galanin in anterior cingulate cortex in rats with acute inflammation. Neurosci Lett. 2017;638:156-161.

8. Vogt BA. Pain and emotion interactions in subregions of the cingulate gyrus. Nat Rev Neurosci. 2005;6:533-544.

9. Vogt BA, Wiley RG, Jensen EL. Localization of Mu and delta opioid receptors to anterior cingulate afferents and projection neurons and input/output model of Mu regulation. Exp Neurol. 1995;135:83-92.

10. Thompson GL, Kelly E, Christopoulos A, Canals M. Novel GPCR paradigms at the $\mu$-opioid receptor. $\mathrm{Br} \mathrm{J}$ Pharmacol. 2015;172:287-296. 
11. Zheng W. Activation of mu opioid receptor inhibits the excitatory glutatergic transmission in the anterior cingulate cortex of the rats with peripheral inflammation. Eur J Pharmacol. 2010;628:91-95.

12. Zimmermann M. Ethical guidelines for investigations of experimental pain in conscious animals. Pain. 1983;16:109-110.

13. Paxinos G, Watson C. The rat brain in stereotaxic coordinates. (fourthed), Academic Press, Sydney. 1998.

14. Li JJ, Zhou X, Yu LC. Involvement of neuropeptide $Y$ and Y1 receptor in antinociception in the arcuate nucleus of hypothalamus, an immunohistochemical and pharmacological study in intact rats and rats with inflammation. Pain. 2005;118:232-242.

15. Jin WY, Liu Z, Liu D, Yu LC. Antinociceptive effects of galanin in the central nucleus of amygdala of rats, an involvement of opioid receptors. Brain Res. 2010;1320:16-21.

16. Li SY, Huo ML, Wu XY, Huang YQ, Wang L. Involvement of galanin and galanin receptor 1 in nociceptive modulation in the central nucleus of amygdala in normal and neuropathic rats. Sci Rep. 2017;7:15317.

17. Zhang ML, Wang HB, Fu FH, Yu LC. Involvement of galanin and galanin receptor 2 in nociceptive modulation in anterior cingulate cortex of normal rats and rats with mononeuropathy. Sci Rep. 2017;7:45930.

18. Livak KJ, Schmittgen TD. Analysis of relative gene expression data using real-time quantitative PCR and the 2(-delta delta C(T)) method, Methods. 2001;25:402-408.

19. Koga K, Shimoyama S, Yamada A, Furukawa T, Nikaido Y. Chronic inflammatory pain induced GABAergic synaptic plasticity in the adult mouse anterior cingulate cortex. Mol Pain. 2018;14:1744806918783478.

20. Thibault K, Lin WK, Rancillac A, Fan M, Snollaerts T. BDNFdependent plasticity induced by peripheral inflammation in the primary sensory and the cingulate cortex triggers cold allodynia and reveals a major role for endogenous BDNF as a tuner of the affective aspect of pain. J Neurosci. 2014;34:14739-14751.

21. Navratilova E, Xie JY, Meske D, Qu C, Morimura K. Endogenous opioid activity in the anterior cingulate cortex is required for relief of pain. J Neurosci. 2015;35:7264-7271.

22. Lucia H, Adrianne WP, Yolanda CJ, Elaine Z, Jose GR. Inflammatory pain promotes increased opioid selfadministration: Role of Dysregulated Ventral Tegmental Area $\mu$ Opioid Receptors. J Neurosci. 2015;35:12217-12231. 